\title{
Transmission dynamics of the ongoing chikungunya outbreak in Central Italy: from coastal areas to the metropolitan city of Rome, summer 2017
}

\author{
Mattia Manica ${ }^{1,2,3}$, Giorgio Guzzetta ${ }^{3,4}$, Piero Poletti ${ }^{3,4}$, Federico Filipponi², Angelo Solimini2, Beniamino Caputo², Alessandra \\ della Torre ${ }^{2}$, Roberto Rosà1, Stefano Merler ${ }^{4}$ \\ 1. Dipartimento di Biodiversità ed Ecologia Molecolare/Centro Ricerca e Innovazione, Fondazione Edmund Mach, San Michele \\ all'Adige, Italy \\ 2. Dipartimento di Sanitá Pubblica e Malattie Infettive, Sapienza University of Rome, Laboratory affiliated to Istituto Pasteur \\ Italia - Fondazione Cenci Bolognetti \\ 3. These authors contributed equally to the work \\ 4. Center for Information Technology, Fondazione Bruno Kessler, Trento, Italy
}

Correspondence: Stefano Merler (merler@fbk.eu)

Manica Mattia, Guzzetta Giorgio, Poletti Piero, Filipponi Federico, Solimini Angelo, Caputo Beniamino, della Torre Alessandra, Rosà Roberto, Merler Stefano. Transmission dynamics of the ongoing chikungunya outbreak in Central Italy: from coastal areas to the metropolitan city of Rome, summer 2017 . Euro Surveill. 2017;22(44):pii=17-00685. https://doi.org/10.2807/1560-7917.ES.2017.22.44-17-00685

\begin{abstract}
A large chikungunya outbreak is ongoing in Italy, with a main cluster in the Anzio coastal municipality. With preliminary epidemiological data, and a transmission model using mosquito abundance and biting rates, we estimated the basic reproduction number $R_{0}$ at 2.07 (95\% credible interval: 1.47-2.59) and the first case importation between 21 May and 18 June 2017. Outbreak risk was higher in coastal/rural sites than urban ones. Novel transmission foci could occur up to mid-November.
\end{abstract}

On 7 September 2017, Italian public health authorities reported three autochthonous cases of chikungunya in Anzio, a coastal city $50 \mathrm{~km}$ south of Rome, located in the Lazio region [1]. However, the symptom onset for the first cases was retrospectively considered to have occurred between 26 and 27 June. The outbreak continued spreading in the Lazio region with the number of notified cases reaching 297 (of which 170 were confirmed) on 13 October. Although most cases were reported from Anzio, a distinct cluster of transmission was also detected in the metropolitan area of Rome [2]. The index case has not been identified, but the mosquito vector implicated in the chikungunya virus (CHIKV) transmission was confirmed to be Aedes albopictus, as was the case in a previous Italian CHIKV outbreak, which occurred in the region of Emilia Romagna in 2007 [1]. In the same period than the Lazio outbreak in 2017, a further outbreak was detected in Guardavalle Marina, a small coastal town in the Calabria region [2], $600 \mathrm{~km}$ south of Anzio, with 54 additional notified cases (nine confirmed). It is still unknown whether the Guardavalle outbreak is epidemiologically linked to the epidemic occurring in Lazio. Here, we provide a quantitative characterisation of the ongoing outbreak, using available epidemiological data [2] and a transmission dynamics model [3-5] informed with data on mosquito abundance [6] and biting rate on humans [7] from previous collections in 18 sites within Lazio region.

\section{Reproduction numbers from epidemiological data}

The instantaneous reproduction number Rt [8] was estimated from the time series of notified cases in Anzio, Rome and Guardavalle Marina under the assumption of gamma distributed generation time ( (shape $=4.67$; scale $=3$; mean $=4$ days) [9] (Figure 1). By averaging Rt over the first 3 weeks of August (initial period of exponential growth), we estimated the basic reproduction number Ro for Anzio at 2.07 (95\% credible interval (Cl): 1.47-2.59), a value slightly lower than that estimated for the 2007 outbreak in Emilia Romagna (i.e. $\mathrm{R}_{0}=3.3 ; 95 \% \mathrm{Cl}$ : 1.8-6.0) [3]. The decrease in $R_{t}$ corresponded with the first date of reactive vector control interventions, namely 7 September [10]. The robustness of this estimate was confirmed by computing the basic reproduction number from the exponential growth rate [11] yielding a very similar result $\left(R_{0}=1.88 ; 95 \% \mathrm{Cl}: 1.55-2.27\right)$. The hypothesis of sub-exponential growth in Anzio was subsequently ruled out [12]. For Rome and Guardavalle Marina, the number of cases was too small to compute a reliable estimate of $R_{0}$; however, peak values of $R_{t}$ for these two outbreaks were smaller compared with the Anzio outbreak (Figure 1).

\section{Mosquito abundance}

We calibrated a mosquito population model [4] to Ae. albopictus capture data obtained at several time points throughout the period July to November 2012 


\section{FIGURE 1}

Time series of notified chikungunya cases with estimates of the instantaneous reproductive number Rt over time, averaged over a moving window of 14 days, Anzio, Rome and Guardavalle Marina, Italy, 2017

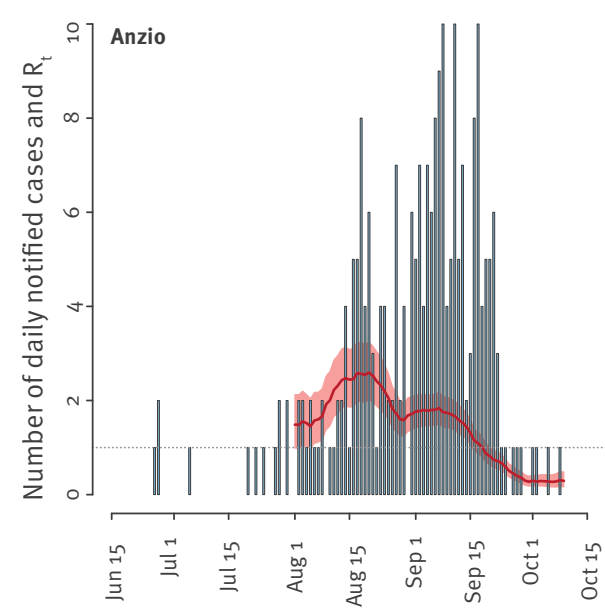

Time (days)

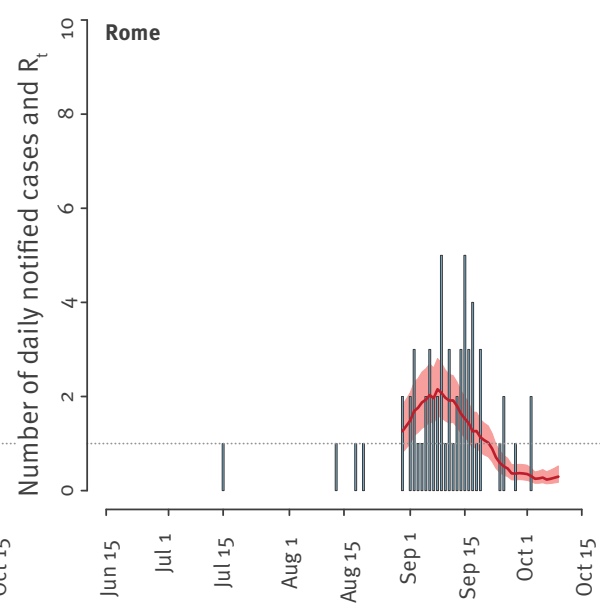

Time (days)

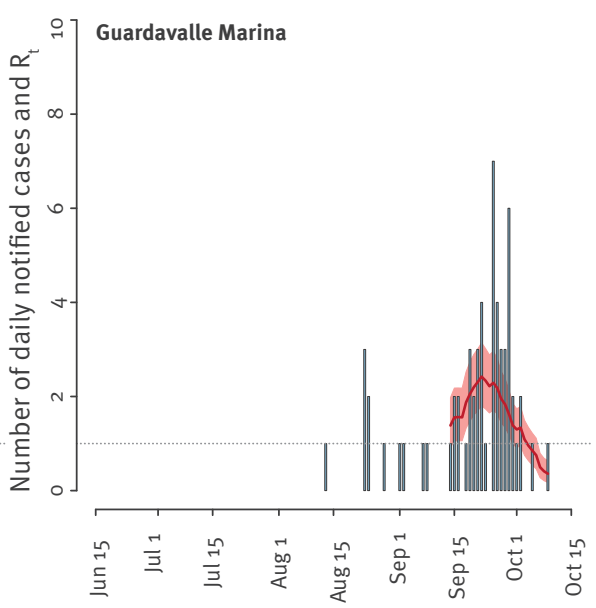

Time (days)

$\square$ Notified chikungunya cases

Estimated instantaneous reproductive number Rt with $95 \% \mathrm{Cl}$

$\mathrm{Cl}$ : credible interval; Rt: instantaneous reproduction number.

Rt was estimated by Markov chain Monte Carlo applied to the Poisson likelihood associated to the renewal equation $\mathrm{Ct}=\mathrm{Pois}(\mathrm{Rt} \Sigma \mathrm{s}=1 \mathrm{tTgsC}(\mathrm{t}-\mathrm{s})$ ) [8], where $C(\mathrm{t})$ is the number of new cases at time $\mathrm{t}$ and $\mathrm{Tg}$ is the generation time distribution [9].

\section{FIGURE 2}

Location within the Lazio region of sites from which mosquito sampling in 2012 provided data for estimation of mosquito abundance in 2017, Italy ( $\mathrm{n}=18$ sites)

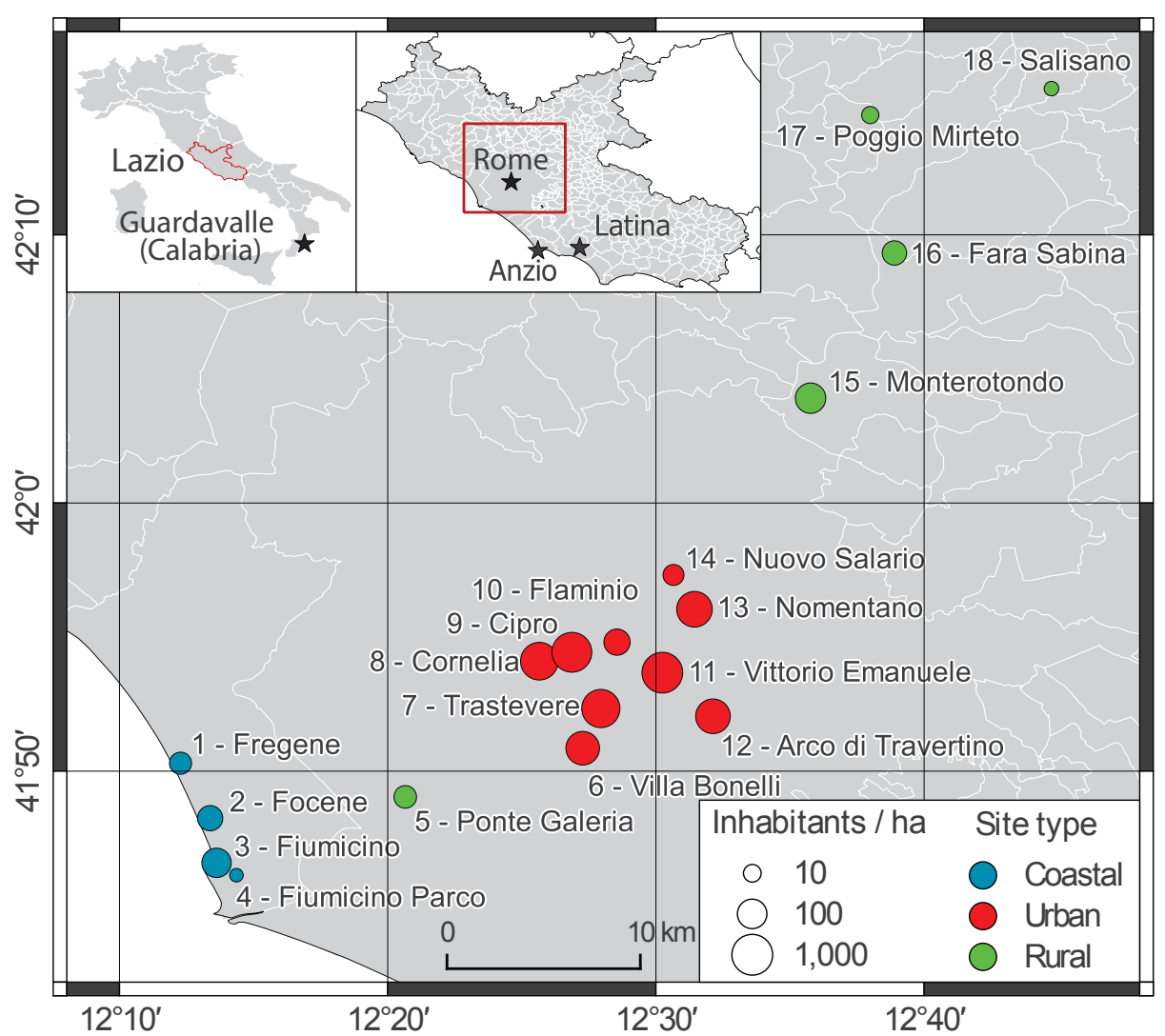

Stars represent locations with ongoing outbreaks in 2017 in Italy. 


\section{FIGURE 3}

Number of Aedes albopictus adult females per hectare over time, as estimated in the absence of interventions for 2017 in the 18 mosquito sampling sites, Lazio region, Italy
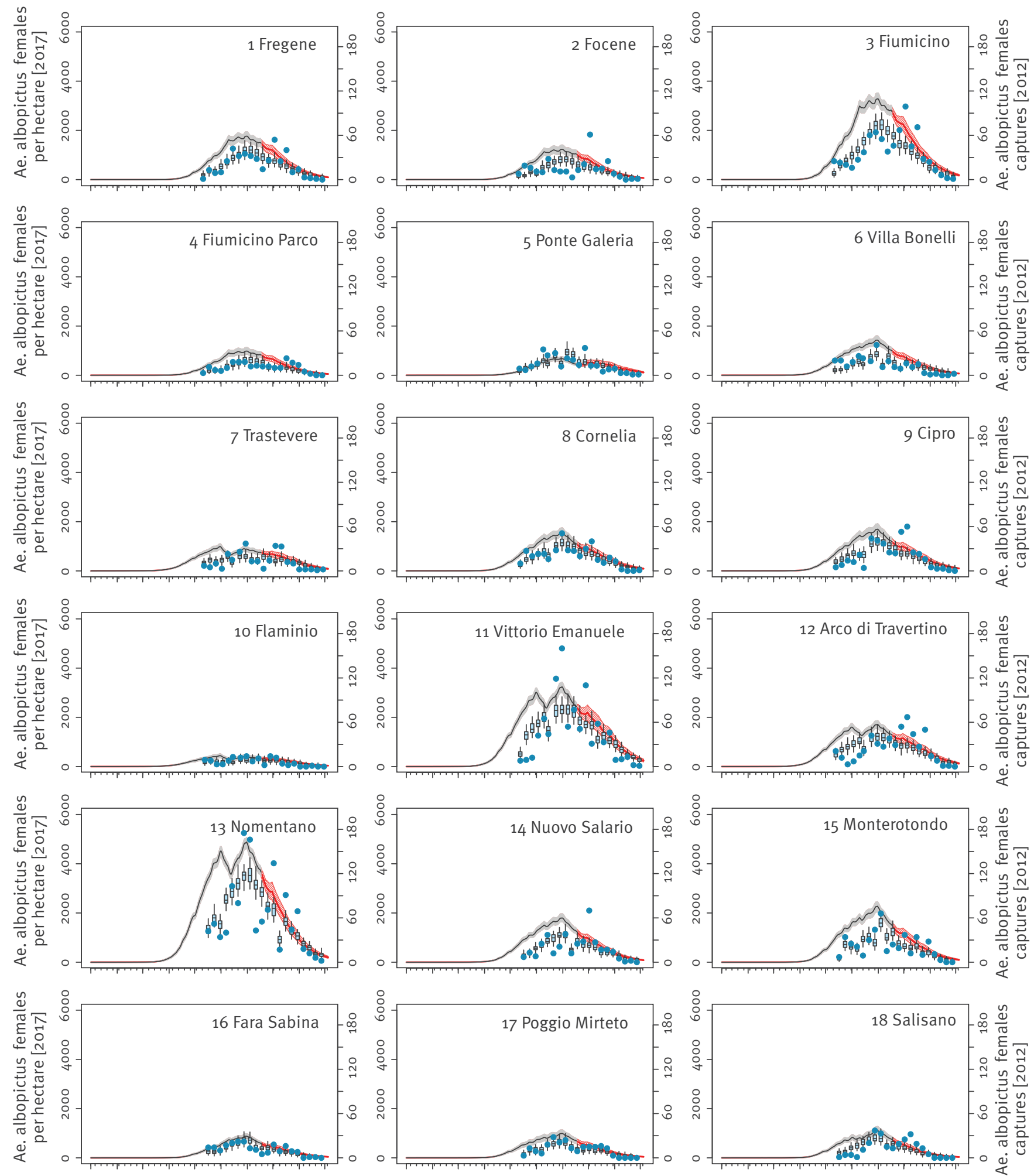

हो.

Time (weeks)

हैं Time (weeks)

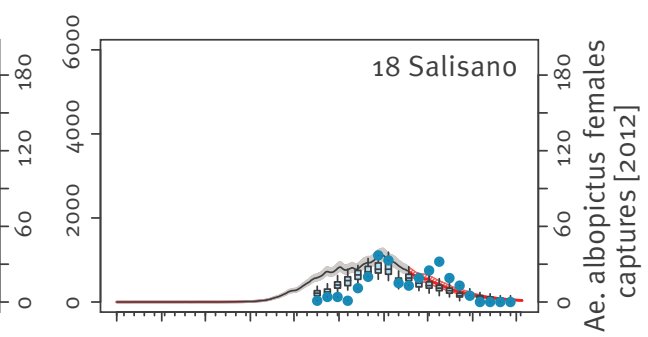

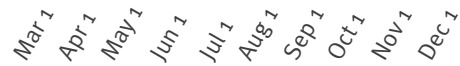
Time (weeks)

For each study site, the abundance of Aedes albopictus adult females per hectare in 2017 is presented over the March to December period (line: mean number; shaded area: $95 \%$ credible interval); the grey colour is used to depict estimates based on recorded temperatures [13], while red is used for estimates from predicted temperatures based on previously observed trends (scale on the left). 


\section{FIGURE 4}

Schematic representation of the model used to estimate chikungunya transmission, Lazio region, Italy, 2017

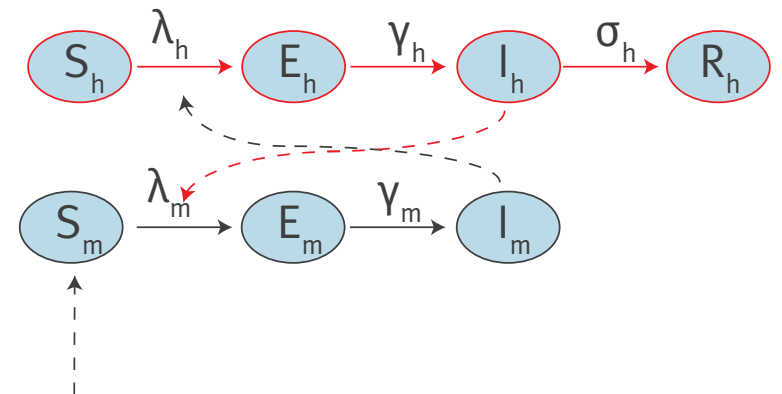

Estimates from observed captures and entomological model

\section{$\mathrm{V}_{\mathrm{h}}:$ Intrinsic incubation period \\ $\sigma_{\mathrm{h}}$ : Human infectious period \\ $\mathrm{V}_{\mathrm{m}}$ : Extrinsic incubation period \\ $x_{m}$ : Probability of human-to-vector transmission per bite}

$x_{h}$ : Probability of vector-to-human transmission per bite

$\mathrm{k}$ : Mosquito biting rate

$\lambda_{m}=k c_{m} I_{h} / N_{h}$

$\lambda_{h}=k c_{h} l_{m} / N_{h}$

$E$ : exposed; I: infectious; $\lambda$ : force of infection, i.e. the probability per unit of time for a susceptible to become infected; N: total population; R: recovered; S: susceptibles.

Subscripts $h$ and $m$ refer to humans and mosquitoes respectively. Human cases are notified with probability pspn, which represent the probability of developing clinical symptoms and the probability of being detected respectively, with a delay $\mathrm{d}$ between symptom onset and detection. Parameters values are reported in the Table.

from 18 sites along a $70 \mathrm{~km}$-transect from the Lazio coast (four sites) to rural inland areas (5 sites), and encompassing the metropolitan area of Rome (nine sites) [6] (Figure 2). Coastal sites have a human density (5-50 inhabitants/ha) close to that of Anzio (roughly 30 inhabitants/ha, increasing during summer months due to touristic influx) and similar eco-climatic conditions, and were therefore considered representative for the analysis of the main outbreak; urban sites (with human density up to 267 inhabitants/ha) were considered representative for the Rome outbreak. The model takes as input daily temperature records obtained from the closest weather station to each sampling site [13].

The calibrated model was re-run with 2017 temperatures to estimate the mosquito abundance during the ongoing outbreak (Figure 3). Human landing capture experiments performed in 2014 within a highly Ae. albopictus infested area in Rome [7] were used to estimate the mosquito biting rate [14]. Remarkably, the biting rate was found to be nearly constant over the season and its value (range: $0.08-$ 0.1 , as shown in the Table) complies with the 0.09 (95\% Cl: $0.05-0.16$ ) estimate from the 2007 CHIKV outbreak $[3,14]$.

\section{Transmission dynamics}

The probability of a CHIKV outbreak, the number of symptomatic and asymptomatic cases and the daily number of notified cases at different sites were computed using a previously published stochastic transmission model [5] (Figure 4) simulated over an area of radius $300 \mathrm{~m}$ (i.e. ca $28 \mathrm{ha}$ ), according to mosquito abundance data [6], epidemiological data [10] and mosquitoes flight range [15]. Potential delays between symptom onset and notification were also accounted for (Table). A set of 10,000 model simulations was run for each site by sampling epidemiological parameters from known distributions and considering a single imported case at different times within the 1 May-15 November time window (Table). In order to predict the time of virus introduction, the symptom onset for the first notified case was considered to have occurred between 23 and 29 June in coastal sites (first recorded symptoms in Anzio: 26 June [2]) and between 12 and 18 July in urban sites (first recorded symptoms in Rome: 15 July [2]). The likely time of virus introduction was identified by selecting simulations with compliant symptom onsets.

According to model estimates, the first CHIKV case is likely to have been imported in the first week of June in Anzio (range: 21 May-18 June, sites 1-4 in Figure 5) and in early July in Rome (range: 28 May-16 July, sites 7-14 in Figure 5). In early June the probability of occurrence of an outbreak is estimated to be higher in coastal sites (11-44\%) compared with urban sites (3-34\%) (Figure 6). However, in the latter sites, the probability of outbreak increases to $22-82 \%$ at the predicted time of arrival of the infection in Rome. The risk of large outbreaks is estimated to be higher in coastal and rural sites than in urban sites (Figure 6), despite the high Ae. albopictus abundance in some urban areas (Figure 2). This is explained by the higher human density in urban sites, which reduces the mosquito/human ratio and thus the risk of infection. Specifically, at the predicted time of the first case in Anzio, the number of mosquitoes per person ranged between 1.9 and 7.3 in coastal sites and between 0.4 and 2.6 in urban areas. The probability of observing additional transmission foci in unaffected areas is estimated to remain significant up to mid-November. This analysis was not performed for Guardavalle Marina due to the lack of entomological data.

\section{Estimates of health and economic burden}

Based on observed cases that occurred before the restriction of blood donations in Lazio on 12 September [1], the estimated time of virus introduction, the notification rates (Table), the durations of infection (Table) and the available estimates on the daily blood donation rates [16], we estimated the probability that one blood sample might have been collected from an infected individual to be ca $0.73 \%(95 \% \mathrm{Cl}$ : $0.28-1.34 \%)$ in Anzio and 0.15\% (95\% Cl: 0.05-0.29\%) in Rome. Based on average costs and Disability Adjusted Life Years (DALY) lost per observed symptomatic CHIKV case [5], 
Distributions of the probable time of first chikungunya virus introduction in coastal sites (sites from 1 to 4 ), which were considered as representative of Anzio, and in urban sites considered as representative of Rome (sites from 7 to 14), Italy 2017
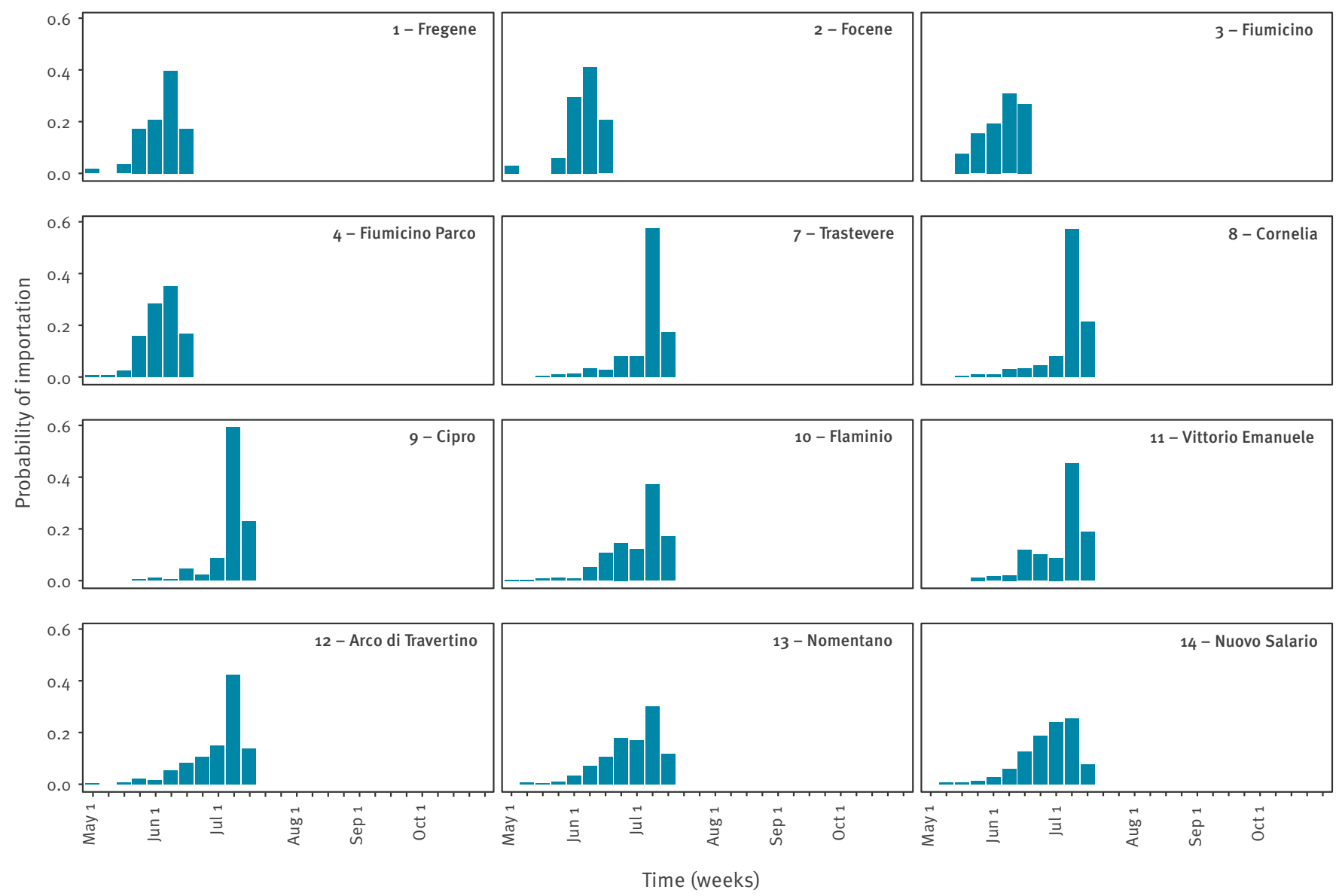

the economic burden as at 13 October is estimated at $322,000$ EUR ( $95 \% \mathrm{Cl}: 222,000-477,000)$ with a loss of 341 DALYs (95\% Cl: 235-505). These estimates exclude costs related to the management of blood supplies after restrictions.

\section{Discussion}

Our modelling estimates are subjected to uncertainties related to the actual mosquito abundance in Anzio and to the provisional nature of epidemiological data available up to now, including possible changes in the detection rates after the outbreak identification. Furthermore, the model is not suitable to evaluate the potential geographical spread of the epidemic, as it provides estimates only at the scale of 30 ha-patches, with the assumption of homogenous mixing within the patch. Critically, the high spatial heterogeneity in mosquito abundance, especially in urban areas, suggests the need to rely on information about mosquito populations at the local scale in order to assess the impact of current and future outbreaks. As shown by past surveillance records $[17,18]$, the number of imported chikungunya cases in Lazio range from zero to seven per year, therefore suggesting that multiple importations from abroad in the city of Anzio during the summer of 2017 were unlikely; however, multiple introductions in
Rome (e.g. infected tourists coming back from Anzio) are possible. This is a further possible limitation to the interpretation of results related to Rome.

Despite these limitations, the model provides relevant estimates to characterise the ongoing CHIKV outbreak in Central Italy. First, the $R_{0}$ in Anzio is shown to be lower, but comparable to $\mathrm{R}_{\mathrm{o}}$ associated with the 2007 CHIKV outbreak in Emilia Romagna and other outbreaks worldwide [3]. Second, perhaps counter-intuitively, the highest transmission potential is predicted in coastal and rural areas (due to the higher mosquito to human ratio compared with densely populated metropolitan areas), consistently with the higher incidence of CHIKV observed in Anzio compared with Rome [2]. Third, the model estimates the health and economic burden related to the outbreak, which are instrumental to evaluate cost-benefits of preventive interventions aimed to reduce mosquito vector densities. In fact, availability of information on insecticide treatments carried out after CHIKV notifications would also allow predicting their effect on mosquito population dynamics. Finally, the model predicts a risk of autochthonous transmission in Lazio region up to mid-November, as a consequence of the expected persistence of favourable climatic conditions in the area [6]. Although the 


\section{FIGURE 6}

Model estimates of the probability of autochthonous transmission of chikungunya virus in 18 mosquito sampling sites in Lazio region, disaggregated by potential outbreak size, in case of a single imported case at different weeks of the year from 1 May to 15 November, Italy 2017
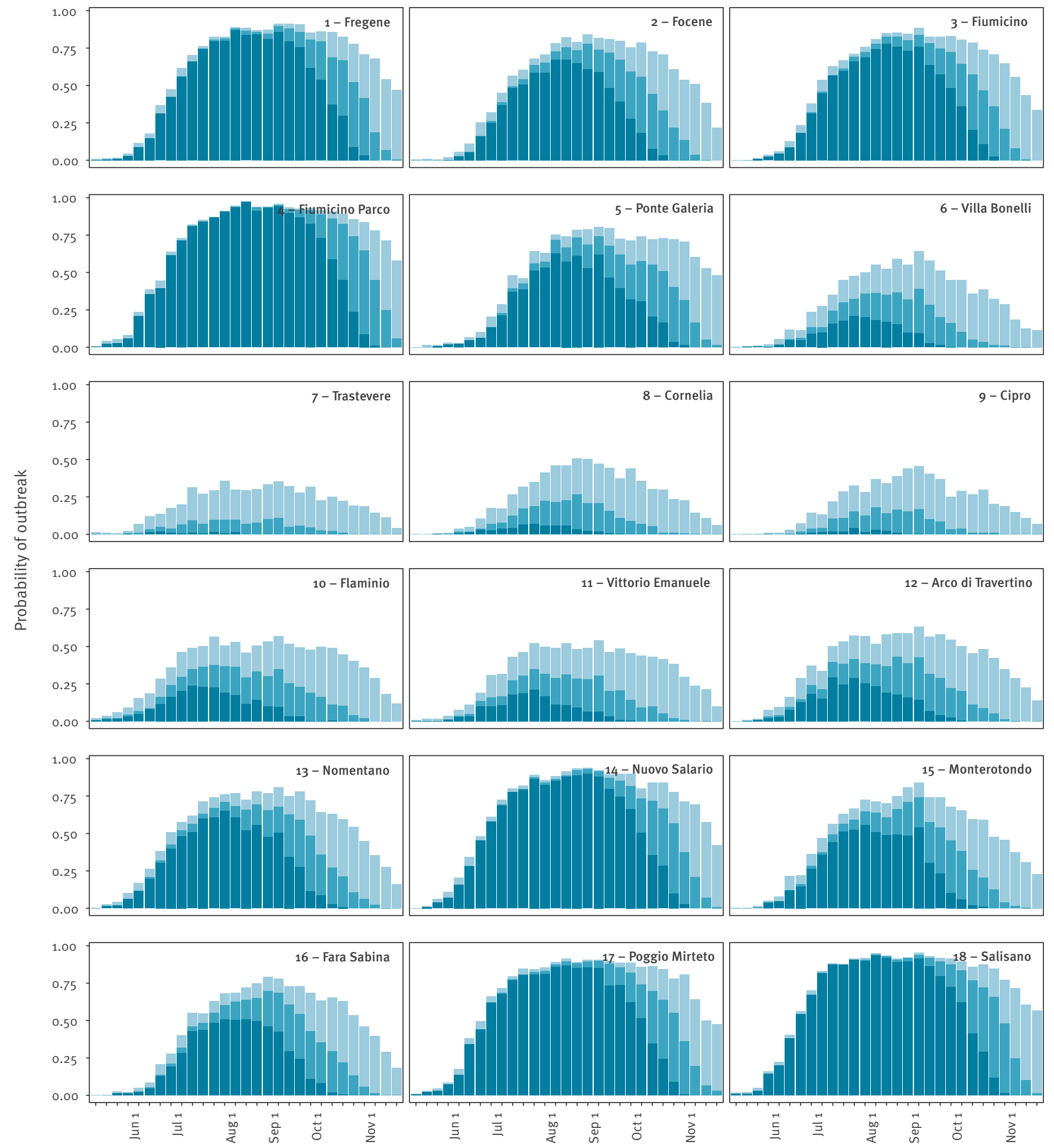

Outbreak size $\quad 2-10 \quad 11-100 \quad>100$

different weeks of the year from 1 May to 15 November, Italy 2017

Figure 6

Click to view

Estimates do not account for the different probabilities of importation (which depend on the absolute number of infected travellers) in urban, rural and coastal sites. 
Epidemiological parameters used in the estimation of transmission in an outbreak of chikungunya in Central Italy, 2017

\begin{tabular}{|c|c|c|c|c|}
\hline Parameter & Unit & Distribution & $\begin{array}{l}\text { Min and } \max ^{a} \\
\text { parameter value }\end{array}$ & Reference \\
\hline Date of imported infection & Date & Uniform & 1 May; 15 Nov & NA \\
\hline Mosquito biting rate & Bites/mosquito/day & Uniform & $0.08 ; 0.10$ & $\begin{array}{c}\text { Own estimate from } \\
\text { [7] } \\
\end{array}$ \\
\hline $\begin{array}{l}\text { Probability of vector-to-human transmission } \\
\text { per bite }\end{array}$ & $\%$ & Uniform & $14 ; 84$ & [21] \\
\hline $\begin{array}{l}\text { Probability of human-to-vector transmission } \\
\text { per bite }\end{array}$ & $\%$ & Uniform & $75 ; 90$ & [22] \\
\hline Extrinsic incubation period & Days & Uniform & $2 ; 3$ & [23] \\
\hline Intrinsic incubation period & Days & Uniform & $1 ; 12$ & [24] \\
\hline Human infectious period & Days & Uniform & $2 ; 7$ & [24] \\
\hline Probability of developing symptoms & $\%$ & Uniform & $65 ; 93$ & [25] \\
\hline Probability of being detected & $\%$ & Uniform & $44 ; 80$ & [25] \\
\hline Delay between symptom onset and detection & Days & Gamma & Scale: 8.53 ; shape: 1.725 & $\begin{array}{c}\text { Own estimate from } \\
{[26]}\end{array}$ \\
\hline
\end{tabular}

Max: maximum; min: minimum; NA: not applicable.

a Unless otherwise specified.

number of cases is declining [19], with only 23 cases notified in October 2017, the foci of CHIKV transmission identified in the city of Latina (22 km east of Anzio) [20] and in Guardavalle Marina highlight the need to continue monitoring the outbreaks.

\section{Conflict of interest}

None declared.

\section{Authors' contributions}

Conceived of the study: MM, GG, PP, RR, SM; Provided/collected the data: MM, FF, AS, BC, AdT; Performed the analysis: MM, GG, PP, SM; Interpreted the results: MM, GG, PP, FF, AS, BC, AdT, RR, SM; Wrote the manuscript: GG, PP, AdT, $\mathrm{SM}$; Revised/approved the manuscript: MM, GG, PP, FF, AS, BC, AdT, RR, SM.

\section{References}

1. Venturi G, Di Luca M, Fortuna C, Remoli ME, Riccardo F, Severini F, et al. Detection of a chikungunya outbreak in Central Italy, August to September 2017. Euro Surveill. 2017;22(39):1700646. https://doi.org/10.2807/1560-7917. ES.2017.22.39.17-00646 PMID: 29019306

2. Istituto Superiore di Sanita (ISS). Bulletin of the National Plan of surveillance and response to arbovirus transmitted by mosquitoes (Aedes sp.), with particular reference to Chikungunya, Dengue and Zika viruses - 2017. Rome: ISS. Italian. [Accessed 19 Oct 2017]. Available from: http:// www.salastampa.salute.gov.it/portale/temi/documenti/ chikungunya/bollettino_chikungunya_20171013.pdf

3. Poletti P, Messeri G, Ajelli M, Vallorani R, Rizzo C, Merler S. Transmission potential of chikungunya virus and control measures: the case of Italy. PLoS One. 2011;6(5):e18860. https://doi.org/10.1371/journal.pone.0018860 PMID: 21559329

4. Guzzetta G, Montarsi F, Baldacchino FA, Metz M, Capelli G, Rizzoli A, et al. Potential risk of dengue and chikungunya outbreaks in northern Italy based on a population model of Aedes albopictus (Diptera: Culicidae). PLoS Negl Trop Dis. 2016;10(6):eoo04762. https://doi.org/10.1371/journal. pntd.0004762 PMID: 27304211

5. Guzzetta G, Trentini F, Poletti P, Baldacchino FA, Montarsi F, Capelli G, et al. Effectiveness and economic assessment of routine larviciding for prevention of chikungunya and dengue in temperate urban settings in Europe. PLoS Negl Trop Dis. 2017;11(9):eo005918. https://doi.org/10.1371/journal. pntd.0005918 PMID: 28892499

6. Manica M, Filipponi F, D’Alessandro A, Screti A, Neteler $M$, Rosà R, et al. Spatial and Temporal Hot Spots of Aedes albopictus Abundance inside and outside a South European Metropolitan Area. PLoS Negl Trop Dis. 2016;10(6):e0o04758. https://doi.org/10.1371/journal.pntd.0004758 PMID: 27333276

7. Manica M, Rosà R, Della Torre A, Caputo B. From eggs to bites: do ovitrap data provide reliable estimates of Aedes albopictus biting females? Peer]. 2017;5:e2998. https://doi.org/10.7717/ peerj.2998 PMID: 28321362

8. Ajelli M, Merler S, Fumanelli L, Pastore Y Piontti A, Dean NE, Longini IM Jr, et al. Spatiotemporal dynamics of the Ebola epidemic in Guinea and implications for vaccination and disease elimination: a computational modeling analysis. BMC Med. 2016;14(1):130. https://doi.org/10.1186/s12916-0160678-3 PMID: 27600737

9. Salje H, Lessler J, Paul KK, Azman AS, Rahman MW, Rahman $M$, et al. How social structures, space, and behaviors shape the spread of infectious diseases using chikungunya as a case study. Proc Natl Acad Sci USA. 2016;113(47):13420-5. https:// doi.org/10.1073/pnas.1611391113 PMID: 27821727

10. European Centre for Disease Prevention and Control (ECDC). Clusters of autochthonous chikungunya cases in Italy. ECDC Rapid Risk Assessment. Stockholm: ECDC; 14 September 2017. [Accessed 4 Oct 2017] Available from: https://ecdc.europa. eu/en/publications-data/rapid-risk-assessment-clustersautochthonous-chikungunya-cases-italy

11. Wallinga J, Lipsitch M. How generation intervals shape the relationship between growth rates and reproductive numbers. Proc Biol Sci. 2007;274(1609):599-604. https://doi. org/10.1098/rspb.2006.3754 PMID: 17476782

12. Chowell G, Viboud C, Simonsen L, Moghadas SM. Characterizing the reproduction number of epidemics with early subexponential growth dynamics. J R Soc Interface. 2016;13(123):20160659. https://doi.org/10.1098/ rsif.2016.0659 PMID: 27707909

13. Regione Lazio, Ufficio Idrografico e Mareografico. [Accessed 4 Oct 2017]. Available from: http://www.idrografico.roma.it/ annali/

14. Guzzetta G, Poletti P, Montarsi F, Baldacchino F, Capelli G, Rizzoli A, et al. Assessing the potential risk of Zika virus epidemics in temperate areas with established Aedes 
albopictus populations. Euro Surveill. 2016;21(15):30199. https://doi.org/10.2807/1560-7917.ES.2016.21.15.30199 PMID: 27104366

15. Marini F, Caputo B, Pombi M, Tarsitani G, della Torre A. Study of Aedes albopictus dispersal in Rome, Italy, using sticky traps in mark-release-recapture experiments. Med Vet Entomol. 2010;24(4):361-8. https://doi.org/10.1111/j.13652915.2010.00898.x PMID: 20666995

16. Grazzini G. Italian policies for blood and blood product selfsufficiency and safety. In Management of Best Standards and Practices for Safe Blood Donation and Transfusion. San Marino; 23 June, 2012.

17. Ministero della Salute. Piano Nazionale di sorveglianza e risposta alle arbovirosi trasmesse da zanzare (Aedes sp.) con particolare riferimento ai virus Chikungunya, Dengue e Zika 2017. Rome: Ministero della Salute; 2017.

18. Ministero della Salute. Piano Nazionale di sorveglianza e risposta alle arbovirosi trasmesse da zanzare (Aedes sp.) con particolare riferimento ai virus Chikungunya, Dengue e Zika 2015. Rome: Ministero della Salute; 2015.

19. Istituto Superiore di Sanita (ISS). Italia: focolai autoctoni di infezione da virus chikungunya. [Italy: autochtonous cases of chikungunya virus]. Rome: ISS; 27 Oct 2017. Italian. Available from: http://www.salastampa.salute.gov.it/portale/temi/ documenti/chikungunya/bollettino_chikungunya_20171027.pdf

20. Regione Lazio. [Accessed 4 Oct 2017]. Available from: http:// www.regione.lazio.it/rl_main/?vw=newsDettaglio\&id=4118

21. Vega-Rua A, Zouache K, Caro V, Diancourt L, Delaunay P, Grandadam M, et al. High efficiency of temperate Aedes albopictus to transmit chikungunya and dengue viruses in the Southeast of France. PLoS One. 2013;8(3):e59716. https://doi. org/10.1371/journal.pone.0059716 PMID: 23527259

22. Talbalaghi A, Moutailler S, Vazeille M, Failloux AB. Are Aedes albopictus or other mosquito species from northern Italy competent to sustain new arboviral outbreaks? Med Vet Entomol. 2010;24(1):83-7. https://doi.org/10.1111/j.13652915.2009.00853.x PMID: 20377735

23. Dubrulle M, Mousson L, Moutailler S, Vazeille M, Failloux $A B$. Chikungunya virus and Aedes mosquitoes: saliva is infectious as soon as two days after oral infection. PLoS One. 2009;4(6):e5895. https://doi.org/10.1371/journal. pone.0005895 PMID: 19521520

24. Thiberville SD, Moyen N, Dupuis-Maguiraga L, Nougairede A, Gould EA, Roques P, et al. Chikungunya fever: epidemiology, clinical syndrome, pathogenesis and therapy. Antiviral Res. 2013;99(3):345-70. https://doi.org/10.1016/j. antiviral.2013.06.009 PMID: 23811281

25. Moro ML, Gagliotti C, Silvi G, Angelini R, Sambri V, Rezza $G$, et al. Chikungunya Study Group. Chikungunya virus in North-Eastern Italy: a seroprevalence survey. Am J Trop Med Hyg. 2010;82(3):508-11. https://doi.org/10.4269/ ajtmh.2010.09-0322 PMID: 20207883

26. Sochacki T, Jourdain F, Perrin Y, Noel H, Paty MC, de Valk H, et al. Imported chikungunya cases in an area newly colonised by Aedes albopictus: mathematical assessment of the best public health strategy. Euro Surveill. 2016;21(18):30221. https://doi. org/10.2807/1560-7917.ES.2016.21.18.30221 PMID: 27172607

\section{License and copyright}

This is an open-access article distributed under the terms of the Creative Commons Attribution (CC BY 4.0) Licence. You may share and adapt the material, but must give appropriate credit to the source, provide a link to the licence, and indicate if changes were made.

This article is copyright of the authors, 2017. 